\title{
EL PAPEL DE LA ACADEMIA EN LA TENSIÓN ENTRE LAS VIEJAS Y LAS NUEVAS FORMAS DE SER Y ESTAR FRENTE A LAS PANTALLAS
}

\section{THE ROLE OF THE ACADEMY IN THE TENSION BETWEEN OLD PEOPLE AND THE NEW WAYS OF BEING AND BE IN FRONT OF} THE SCREENS

\section{Leandro Arbey Giraldo Henao ${ }^{1}$ Jaime Andrés Ballesteros $^{2}$ Luis Fernando Carrillo Holguín ${ }^{3}$}

Grupo de investigación ACDM. Universidad Tecnológica de Pereira-Colombia

$1 \quad$ Avance de una investigación en curso, titulada Observatorio de análisis del discurso televisivo en el aula: una propuesta integradora y didáctica para la formación de audiencia en la escuela de español y comunicación audiovisual de la universidad tecnológica de Pereira-Colombia (2018 - 2020). Vicerrectoría de Investigaciones de la Universidad Tecnológica de Pereira. El trabajo está financiado en virtud de su aprobación en la convocatoria interna para el desarrollo de proyectos de investigación año 2017. Grupo de Investigación A.C.D.M.

\section{$2 \quad$ lagh@utp.edu.co Cel: 3104949545 https://orcid.org/0000-0002-5345-0801}

Doctor en Ciencias de la Educación. Área pensamiento educativo y comunicación, Universidad Tecnológica de Pereira. Magister en Lingüística, Universidad Tecnológica de Pereira. Licenciado en español y comunicación Audiovisual, Universidad Tecnológica de Pereira. Investigador y profesor asociado, Área de ciencias del Lenguaje, Universidad Tecnológica de Pereira, Facultad de Ciencias de la Educación. Autor de los libros: Forma y sentido de la ciencia del lenguaje. Fundamentos teóricos y prácticos del discurso lingüístico, pedagógico y juvenil (2016); Representaciones discursivas del habla juvenil en la Educación Media (2017). Entropías necesarias. Provocaciones del lenguaje, la filosofía y la literatura para una educación crítica (2018). Ha publicado artículos en revistas especializadas nacionales e internacionales en torno al lenguaje, la comunicación y el discurso; filosofía y lenguaje; lenguaje y educación, lenguaje y literatura. Actualmente se dedica a la investigación en calidad de director del grupo: A.C.D.M (Análisis Crítico del Discurso Multimodal: Estudios y aplicaciones transversales en ámbitos socio-académicos). Pertenece en calidad de co-investigador al grupo de investigación del doctorado en educación. Filosofía pos-metafísica dirigido por el Doctor Julián Serna Arango (Universidad Tecnológica de Pereira). Correo electrónico: lagh@utp.edu.co

3 jaballesteros@utp.edu.co https://orcid.org/0000-0003-2350-4052 Cel: 3185363531 Egresado de la Maestría en Estética y Creación de la Universidad Tecnológica de Pereira. Ingeniero Industrial de la misma Universidad. Se desempeña como profesor catedrático en las asignaturas de Cine y Lenguaje Audiovisual. Gestor Cultural en temáticas de Cultura Ciudadana y Formación de Públicos. Ingeniero Industrial con experiencia en Desarrollo y Administración de procesos de Creación, Gestión y Promoción Cultural. Investigador en Estética y Creación.

\section{$4 \quad I f e r n a n d o c @ u t p . e d u . c o$ Cel. 3206875143}

Egresado de la Maestría en Lingüística de la Universidad Antioquia. Doctor en Ciencias de la Educación, por la Universidad Tecnológica de Pereira (Rudecolombia); magíster en Lingüística y Licenciado en Español y Comunicación Audiovisual, por la Universidad Tecnológica de Pereira. Se desempeña como profesor catedrático en las asignaturas de Lenguaje y Socialización y Análisis del Discurso en el mismo contexto universitario. Sus áreas de investigación son los estudios del discurso didáctico, pedagógico y político. 


\section{RESUMEN}

Este artículo reflexiona sobre el papel de la academia en la tensión entre las viejas y las nuevas formas de ser $y$ estar frente a las pantallas. Se fundamenta teóricamente en dos momentos cruciales que soportan el desarrollo investigativo, cuyos abordajes se conectan con las viejas y las nuevas formas de ser y estar frente a las pantallas. En lo primero, se integra con una perspectiva teórica e histórica de la televisión en el ámbito latinoamericano, $\mathrm{y}$, en lo segundo, con postulados sobre las formas modernas en que los jóvenes estudiantes universitarios se relacionan con la pantalla televisiva, en particular, y las multipantallas, en lo global. Se inscribe en una investigación de tipo cualitativa, con apoyo cuantitativo desde un enfoque descriptivo-interpretativo, en función de una perspectiva pedagógica sobre el desconocimiento de una presencia efectiva de las nuevas formas de ser y estar frente a las pantallas, así como el nivel de implicación de los hábitos y las discursividades propias de los estudiantes objeto de estudio.

\section{PALABRAS CLAVE}

Academia, televisión, pantallas, tensiones, viejas formas de ver televisión, nuevas formas de ver televisión.

\section{ABSTRACT}

This article reflects upon the role of academia in the tension between old and new ways of being in front of screens. Theoretically, it is based on two crucial moments that support research development, whose approaches are connected with old and new ways of being in front of the T.V. screen. Firstly, it is integrated with both a theoretical and a historical perspective of television in the Latin American context, and, secondly, with postulates on modern ways in which young university students relate to the television screen in particular and to multi screens in general. This descriptive-interpretive qualitative research with quantitative support aims at understanding, from a pedagogical perspective, the lack of knowledge regarding an effective presence of new ways of being in front of the screens, as well as the level of involvement of habits and discursiveness of university students under study.

\section{KEY WORDS}

Academia, television, screens, tensions, old ways of watching television, new ways of watching television.

La televisión sigue constituyendo el medio de difusión más importante para las grandes mayorías. Para bien o para mal, a la televisión en general se le dedica, en los países de la región, un mínimo de tres horas diarias entre semanas y cinco o más durante los fines de semana.

La televisión es la opción preferida por los latinoamericanos para disfrutar el tiempo libre $y$, cada vez más, también para pasar ese otro tiempo anteriormente reservado a otras actividades o diversiones. Las horas dedicadas a estar con la familia cada vez más incorporan la televisión o se desarrollan alrededor del televisor.

En las medianas y grandes ciudades latinoamericanas se registra esa tendencia a concentrar en la televisión en entretenimiento, la distracción, la información, la conversación y, en suma, gran parte de toda esa vida que se lleva a cabo "bajo techo".

Guillermo Orozco (2012)

Historia de la televisión en América Latina

\section{INTRODUCCIÓN}

La academia en el marco de las tensiones educativas en la historia de occidente, ha jugado un papel determinante para el desarrollo formativo, social y político. Ya sea a favor de visiones clausuradas o perspectivas 
progresistas, su protagonismo ha sido de gran talante en los procesos de enseñanzaaprendizaje, en donde el hombre ha participado con sus capacidades, intereses, configuraciones intelectuales y desarrollos cognitivos, de acuerdo con las construcciones sociales de las realidades emergentes (Berger \& Luckman, 1968).

En los últimos años, una de las tensiones más recurrentes a las que se ha enfrentado la academia ha sido la incorporación o no de la televisión en el aula de clases (Rincón, 1995). Esta discusión ha hecho que algunos teóricos, docentes y pensadores tradicionales, asuman el hecho como una especie de interferencia en los circuitos de educación, entendidos desde la sinergia entre el docente, los temas educativos, los alumnos y la repetición posterior de los "saberes aprehendidos" por parte de los educandos.

No así ha sido el caso de otros (Orozco, 2012), quienes han asumido el fenómeno tecnológico, en general, y de la televisión, en particular, como una posibilidad de comprender las construcciones sociales de la realidad (Berger \& Luckman, 1968) y las discursividades de los estudiantes en relación con la interpretación, la creación y re-creación del conocimiento; derivados, al parecer, de contenidos televisivos, plataformas universales y de la internet, procurados a través de diferentes medios, modos y multipantallas, entendidos como los nuevos territorios comunicacionales (Luhmann, 1990).

Ha sido frecuente en la academia el hecho de encontrar perspectivas teóricas y modus operandi signados en la reiteración y la tradición para incorporar el "conocimiento" a los estudiantes, como si fueran cosificaciones que se encasillan en formatos discriminados por áreas, disciplinas y momentos particulares, en búsqueda de que sean recitados sin más ni más.

A dichas determinaciones el mismo Freire (1970) Ilamaría una educación bancaria; no muy distante de aquello que en la presente década acontece en muchas de las instituciones de corte academicista y tradicional, que persisten en sus modos de vaciar contenidos. Han sido usanzas educativas altamente metafísicas, cuyos asideros los encontramos en las vías hegemónicas de la razón y el logos a secas, como bien los pensadores clásicos griegos (Sócrates, Platón, Aristóteles) lo determinaron para occidente.

No obstante, en este embate y combate de focalizaciones teóricas, metodologías, métodos y experiencias, otras formas de ver la educación han ido ganando terreno en los nuevos modos de entender las dinámicas pedagógicas para el siglo XXI. En este sentido, cabe señalar que desde Heráclito hasta Nietzsche, la larga noche metafísica instaurada fue cediendo terreno en la medida en que las provocaciones en materia filosófica y educativa de pensadores posteriores al genio alemán, desencadenaron estruendos teóricos y metodológicos para establecer tensiones y contrapunteos en el marco de la formación del hombre en la academia contemporánea.

Justamente, en este horizonte es en el que se desarrolla la presente reflexión, derivada del proyecto de investigación: Observatorio de análisis del discurso televisivo en el aula: una propuesta integradora y didáctica para la formación de audiencia en la escuela de español y comunicación audiovisual de la Universidad Tecnológica de Pereira-Colombia, pensado a partir de las viejas y las nuevas

5 En esta perspectiva se sostiene que han sido el principio de no contradicción en Sócrates, el mundo de las ideas en Platón y la visión del tiempo lineal y los fines (El thelos) en Aristóteles, los que han marcado una academia logocéntrica, distante de las contingencias y del devenir propio del mundo, sus relaciones y construcciones, tal como los pre-socráticos lo sintieron; esto último, en el mundo contemporáneo está naturalmente marcado con la fluidez de la vida, las posibilidades latentes para ser y estar, y el constante cambio de las cosas y los seres humanos; las relaciones con los medios tecnológicos: dispositivos y pantallas, en dichos movimientos naturales, no son la excepción. 
formas de ser y estar frente a las pantallas ${ }^{6}$; en especial, de la televisión y las maneras en las que la comunidad estudiantil, objeto de análisis, se relaciona con la tecnología, las redes sociales y las multipantallas.

Por tanto, en el transcurso de este texto se opta por una visión contrastiva, y se apuesta teóricamente sobre el papel de la academia en esta particular tensión; que, entre otras, constituye una de las reflexiones que animan profundas discusiones en las renovaciones educativas, que van más allá de la crítica, y edifican miradas desde la propia reflexión y práctica pedagógica. No de otro modo, que en función del acompañamiento del educando en una suerte de edificación autónoma (Arboleda, 2016) para el fortalecimiento de sus procesos de formación intelectual en el aula y fuera de ella. En esta línea argumentativa, el autor en referencia define el enfoque edificador en la pedagogía del siguiente modo:

Se trata ésta de una perspectiva ótrica, es decir que promueve y precisa, para su apropiación e implementación, de potenciales tales como disposiciones, capacidades, valores y actitudes para mediar en los procesos de aprendizajes semánticos y comprensivos bajo el mínimo inalienable de acoger al otro en sus necesidades y complejidades, siempre con miras a que los resultados de las acciones formativas eleven en los implicados la calidad de ser humano, de ser mejores personas, artífices de la construcción de mundos humanamente buenos, mejores (Arboleda, 2018:1).

De ahí que el fenómeno del cual se ocupa el estudio parta de la siguiente pregunta y nos permita un primer acercamiento teórico-reflexivo: ¿Cuáles son las prácticas del estudiante de la
Lic. en Comunicación e Informática Educativas, como audiencia televisiva, que pueden enmarcarse dentro de "Las nuevas formas de ser y de estar frente a la pantalla televisiva"?

En tal virtud, el escrito presenta un planteamiento propio sobre lo que podría ser el papel pedagógico de la academia en la tensión entre las viejas y las nuevas formas de ser y estar frente a las pantallas; cimentado, como es natural, en voces teóricas que analizan históricamente las antiguas formas en las que los espectadores se relacionaron con la pantalla chica y su evolución, una vez surge la televisión en Colombia.

Adicionalmente, en la literatura vigente alrededor de las nuevas formas de ser y estar de los jóvenes [estudiantes] frente a las pantallas, cuyos sentidos riñen con visiones académicas centralistas y hegemónicas estiladas aún en la presente centuria; su tradición paquidérmica pretende seguir encapsulando el conocimiento en enfoques logo-céntricos y platónicoaristotélicos, como si las movilidades en los procesos cognitivos no existieran, o como si las detonaciones nietzscheanas no hubieran destapado grandes falacias, auspiciadoras de transformaciones y posibilidades en el devenir del mundo, las cosas, la inventiva humana y las interacciones sociales (Halliday, 1979).

En este panorama, los postulados de teóricos en el campo (Orozco, 1985, 2010; Rincón, 1995; Hall, 1980), constituyen fuentes esenciales para la discusión y el análisis; pues, como se expondrá más adelante, según la teoría recopilada y la discusión, existen nuevas formas de acercarse a la televisión que no solo pugna con las viejas formas de abordarla, usarla y entenderla, sino también, con las posturas de la academia frente a los modos en que deben asumirse tales dinámicas, de cara a los procesos educativos y

$6 \quad$ Con este término nos referimos a los diferentes dispositivos con los cuales los sujetos sociales, en general, y los jóvenes estudiantes de la presente época, en particular, acceden y se relacionan con variados contenidos transmitidos tanto por la programación televisiva en Colombia, como por la programación universal disponible en las múltiples plataformas y sitios web, a los que es posible acceder libremente o a través de pagos de bajo coste. 
formativos del hombre contemporáneo.

Este texto, en consecuencia, transita por caminos que comprenden la educación como un campo de batalla (Zuleta, 1985) que ha virado en sus escenarios, entornos, métodos, estrategias y pedagogías para la lucha educativa. La naturaleza propia de las cosas, de los estados, las relaciones de enseñanza-aprendizaje y el avance del hombre y de la vida en los diferentes ámbitos en los que su ser sentí-pensante ha interactuado, han sido los detonantes para advertir nuevos rumbos y proyecciones sobre posturas y entendimientos divergentes; lo que de fondo nos interesa pedagógicamente, suscrito en la relación: educación-pantallasaprendizajes.

Elanálisis de diferentes poblaciones relacionadas con las viejas y nuevas formas de ser y estar frente a las pantallas, ha sido abordado por autores como: Rincón, (2002); Orozco, 2010; Bolter y Grusin, 2011; Rodríguez, 2012; González, Ruíz y Velásquez, 2012; Ciaramella y Biscuti, 2014; Longwell, 2014; Lazo, 2017; Hernández y Martínez, 2017; etc.). Sin embargo, una vez la revisión de esta literatura, se concluye que estos estudios, aunque pertinentes para la revisión del estado de la cuestión y claves como fundamentos para el análisis, no han abordado el fenómeno a partir de momentos significativos del transcurso de una carrera universitaria relacionada con la televisión en universidades públicas o privadas en el país, que dé cuenta de las prácticas en el marco de las viejas y nuevas formas de ser y estar frente a las pantallas, y que, a su vez, constituya un insumo para los docentes y sus discursividades dentro del aula.

Un caso diferente se pretende con la investigación que suscita este apartado, toda vez que a partir del entendimiento y la interpretación crítica de los fundamentos teóricos, se identifica, categoriza y analiza las prácticas de los estudiantes de la Lic. En comunicación en Informática
Educativas, frente a las pantallas, para aportar a una práctica pedagogía diversa, progresiva y edificadora en aula y fuera de ella; en relación con las discursividades de los estudiantes y la posibilidad de los docentes de la asignatura de televisión en la Universidad Tecnológica de Pereira, para crear y re-crear en el aula, a partir de los insumos entregados.

En suma, las consideraciones expuestas hacia el final del texto esbozan la idea según la cual, las prácticas pedagógicas alejadas de la hegemonía tradicional pueden conformar gran parte del papel edificador que la academia en sus exploraciones pedagógicas, podría asumir en la tensión entre las viejas y las nuevas formas de ser $y$ estar frente a las pantallas de los jóvenes estudiantes de hoy; así, lo planteado, constituye un avance sinérgico de los fundamentos teórico-metodológicos de la investigación en la que se circunscribe el presente acápite.

\section{ASPECTOS METODOLÓGICOS}

Como se indicó arriba, esta reflexión forma parte de un trabajo de investigación con los estudiantes matriculados en el I, VI y X semestres entre agosto 2017 a agosto 2018, de la Lic. En Comunicación e Informática Educativas, programa de la Escuela de español y comunicación Audiovisual de la Universidad Tecnológica de Pereira, que busca proponer sobre el papel de la academia en la tensión ya mencionada. Para ello, se contó con el permiso de la dirección de la escuela, de los estudiantes $y$ de los docentes quienes en su momento dirigían los grupos de la asignatura Televisión. La población fue abordada en cuatro momentos significativos de su avance curricular en la carrera: I semestre, inicio del VI semestre, final del VI semestre, y en el último semestre.

Se aplicó a todos los estudiantes matriculados en cada uno de los cuatro momentos establecidos, con el propósito de que con el 
pasar de los semestres, una vez el observatorio cumpla varios años de estar auscultando las dinámicas frente a la televisión que "llegan" a la Licenciatura, vía los grupos de estudiantes, se pueda decir que toda la población de la carrera fue examinada. La perspectiva metodológica del análisis es cualitativa con apoyo cuantitativo, a partir de un enfoque descriptivo-interpretativo (Deslauriers, 2005; Bernal, 2010).

El método consistió en 1). Aplicar encuesta semiestructurada a los estudiantes objeto de estudio.

2) Conversar con los grupos focales y determinar sentidos alrededor de las viejas y nuevas formas de ser y estar frente a la pantalla, a partir de las narraciones llevadas a cabo. 3) Contrastar los datos obtenidos con los fundamentos teóricos de la investigación. 4) Analizar los resultados a la luz de los lentes teóricos 5) Identificar las prácticas de observancia de la televisión y 6) determinar el insumo general para los docentes y sus discursividades en el aula, para la obtención de prácticas pedagógicas alternativas y edificadoras, en función del papel de la academia en la tensión entre las viejas y las nuevas formas de ser y estar frente a las pantallas.

Llegados a este punto, a continuación se exponen algunos de los referentes teóricos en los que se establece la totalidad de la pesquisa, para concluir con una apuesta puntual relacionada con el papel que podría cumplir la academia, en la tensión entre las viejas y las nuevas formas de ser y estar frente a las pantallas, tal y como se ha venido indicando.

Naturalmente, dejamos claro que es una de las tantas posibilidades que pueden gestionarse a partir de la investigación particular y contextual, en diferentes escenarios educativos de educación superior, donde la observación de las prácticas de los estudiantes frente a las pantallas, constituya el interés de un programa académico específico, para entender el desconocimiento sobre la presencia efectiva de las nuevas formas de ser y estar de los jóvenes frente a la televisión y las multipantallas; y, en ello, los niveles de implicación de los hábitos y sus discursividades.

No alejado, preferiblemente, $y$, como es propio del enfoque filosófico y pedagógico en el que nos situamos, de una mirada abierta, edificadora y plural, sobre las relaciones de enseñanza-aprendizaje; más aún, cuando la nueva demanda del estudiante, la sociedad y las actuales tecnologías de información, conforman un hecho contundente y progresivo en la transformación del orden mundial.

\section{REFERENTES TEÓRICOS}

\subsection{Viejas formas de ser y estar frente a la pantalla de T.V. Perspectiva histórica}

\subsubsection{La televisión: De satélite familiar a satélite de la Escuela}

En un país fraccionado por los permanentes conflictos armados, las minorías se han afincado históricamente en diferentes estrategias de producción y reproducción del poder para mantener su statu quo. Esto ha acontecido desde el mismo momento de su independencia hasta entrado el siglo XXI, en cuyo desarrollo Colombia se ha caracterizado como un país desigual y en permanente guerra, donde el poder económico y político ha estado concentrado en la mano de unos pocos.

En este contexto llegó la televisión al territorio colombiano de la mano de un gobierno militar, que inició labores el 13 junio de 1954 (Rey, 2002:117)

El gobierno del General Gustavo Rojas Pinilla encontró en este nuevo recurso tecnológico

7 Cabe señalar que según Rey (2002: 123), la televisión colombiana se originó como un proyecto de naturaleza estatal asignado al departamento de propaganda de un gobierno militar (la ODIPE, oficina de información y prensa del Estado, dependencia directa de la Presidencia de la República). 
la posibilidad de convocar, a un país dividido, hacia la convivencia y la reconstitución de todos los elementos que permitieran reunificar la patria, luego de turbulentas décadas de conflicto que dejaron una estela de muerte y una enorme brecha entre la sociedad urbana y rural; aunado, como si fuera poco, a una profunda crisis económica que conllevó al endeudamiento del país con gobiernos extranjeros, y en consecuencia, al enorme endeudamiento, sometimiento a poderes transnacionales y a la búsqueda de estrategias y caminos que llevaran a la reconciliación de un pueblo fragmentado.

Así las cosas, el gobierno de turno halló en la televisión la posibilidad de reunificar una nación desde el direccionamiento de una programación televisiva controlada por el Estado y direccionada a partir de contenidos ligados a la simbología patriótica y al fortalecimiento de la cultura elitista. A esto, se le sumó una fuerte inscripción en los valores tradicionales agenciados por la iglesia Católica que, para el momento, después de tener una notable influencia e injerencia en la educación, volcaba su interés en este emergente medio de comunicación masiva.

En un contexto en el que subsistían las diferencias partidistas entre liberales y conservadores, avivadas por profundas realidades de exclusión, la televisión en Colombia entró a participar y a reflejar la realidad del país, $y$, con ello, proyectar los fenómenos migratorios a partir del progresivo traslado de los pobladores del campo a la ciudad; a su vez, del crecimiento desbordado y no planificado, de la creación de nuevos oficios ajustados a estas necesidades y del tránsito de la consolidación de nuevas formas de vida urbana.

Dado lo anterior, la televisión procuró propiciar nuevas oportunidades educativas, participar en la modernización de Estado y de las instituciones, influir en las transformaciones de la familia, en la percepción y valoración de las dinámicas que se surtían ante esta nueva configuración de sociedad neo-urbana, como apreciaciones de autoridad, renovaciones estéticas, variaciones de la moda, etc. Es decir, la televisión en su injerencia directa en los gustos, preferencias colectivas y contrastes culturales (Rey, 2002: 119-120).

SibienllegódelamanodelEstadopredeterminada como una estrategia de control y consolidación de un poder, en un país manejado por familias ricas y con intereses económicos claros, muy pronto la programación de la televisión dejó al descubierto, más allá de su notable rol de ejercicio de poder, una oportunidad de amasar fortuna y ejercer una influencia ideológica y política. No gratuito, Rincón (1995: 13), señala que: "La radio y la televisión son consideradas como los medios clásicos de comunicación, por tal razón siempre se han encontrado en el centro del debate social, político y educativo".

Derivado de esta dinámica, es posible señalar cuatro tendencias o momentos (Rey, 2002: 120), mediante los cuales caracterizar el desarrollo de la televisión en el país de los más grandes contrastes a través de la historia, con el ánimo de consolidar nuestra perspectiva sobre el papel de la academia en la tensión entre las viejas $y$ las nuevas formas de ser y estar frente a las pantallas:

1) En primera instancia, la televisión deja de tener un carácter cultural y público, para dar paso a la creación de empresas privadas que promueven la producción de programas televisivos producto de la gestión y promoción de grupos económicos que encontraron en este naciente medio, como ya habría ocurrido con la radio, una oportunidad de negocio.

2) Se relativiza el concepto de cultura a partir de las necesidades derivadas de lo comercial, lo cual trae como consecuencia la reorientación del foco televisivo hacia aquellas temáticas y contenidos que gozan de mayor aceptación dentro del público, lo que significa un enorme 
cambio frente a la naturaleza de lo propuesto en esos momentos iniciales cuando los colombianos tuvieron noticia de la naciente televisión.

Queda así sentado que la televisión llegó para quedarse, al ganarse prontamente un espacio muy importante dentro de las actividades cotidianas de los colombianos, asociado a su consolidación como industria la remite necesariamente a su estructuración desde pautas claras que permitieron su funcionamiento y operación dentro de un marco político, económico y jurídico. Es así, como se crean las diversas ofertas televisivas definidas a partir de sus objetivos, orientaciones, financiamiento, reglamentaciones, recursos tecnológicos, cobertura y programación, de los que emergen, entonces, las tres tipologías de canales que a la fecha hacen uso del espectro electromagnético colombiano.

3) Se formaliza la televisión privada en Colombia, con los dos canales que capturan la mayor teleaudiencia del país, RCN y CARACOL TV, con autonomía e independencia en su parrilla de programación, contenidos y orientación de los mismos dentro de un esquema de libre competencia en la que estos canales pujan por capturar la mayor audiencia.

De otro lado, el Estado sigue bajo el control de los canales nacionales públicos a excepción del CANAL UNO. Con un financiamiento totalmente estatal se pretende la realización de programas de interés público ajenos al ánimo de lucro $\mathrm{y}$, finalmente, la consolidación de canales de televisión que responden a necesidades locales, encarnadas en lo que hoy son los diversos canales regionales que funcionan en el país. Al respecto sugiere Rey, que se generan modificaciones estructurales de las tendencias anteriores:

Modificaciones políticas, jurídicas
y económicas que van creando
paulatinamente diversas televisiones,

definidas por sus posibilidades y limitaciones señaladas por las reglamentaciones, o por sus estructuras de propiedad, el uso de las tecnologías, las coberturas o los diseños de programación (Rey, 2002: 120).

4) Una cuarta y última tendencia de la televisión pública en Colombia, la constituye la evolución y consolidación de géneros televisivos que permitieron caracterizar la producción al interior del país y fuera de él. Son representativas de la televisión nacional las telenovelas, los dramatizados, los noticieros y los programas de opinión, al punto que algunos de ellos han logrado romper las fronteras para ser difundidos en otros canales latinoamericanos.

El compendio realizado resumen, tal como lo anota Guillermo Orozco (2012), que la televisión se consolida como el medio de comunicación más importante para la mayoría de los Latinoamericanos, en tanto millones de ellos diariamente dedican varias horas a la televisión, al constituirse en la alternativa más económica, segura y sin compromiso alguno para el disfrute del tiempo libre. La televisión relevó otras prácticas o diversiones hasta llegar a entronizarse en la familia quien la incorporó como una actividad más del diario vivir.

En suma, la televisión encarna el entretenimiento, la distracción y la información desde las cuales han mediado las últimas generaciones, tanto en nuestro país como quizás en el mundo entero. Lo cual, con el tiempo, mutó para pasar de ser el satélite de la familia a ser un controvertido "satélite de la escuela", y constituirse tanto en un objeto de reflexión, como en una herramienta de trabajo didáctico y pedagógico.

No obstante, como se ha expresado desde el inicio, ha significado un elemento de profundas querellas teóricas y académicas, dadas las posturas a favor y en contra que ponen en tensión no solo las viejas y nuevas formas de acercarse 
a la televisión, sino las propias prácticas pedagógicas y las maneras de re-pensar la educación de los sujetos contemporáneos; que, querámoslo o no, ya no hacen parte de realidades cotidianas y educativas caducas, sino, por el contrario, de ánimos diversos, progresivos, interactivos y modos de vida tendientes a la rapidez, el divertimento y la edificación propia de nuevas realidades, en donde su construcción social, pasa por el individuo y sus decisiones en la vida cotidiana, ubicadas habitualmente más allá de la Escuela; las nuevas formas de ser y estar frente a las pantallas hacen parte de aquellas.

He aquí el punto neurálgico en cuyos desafíos el papel de la academia en la tensión entre lo pasado y lo contemporáneo es determinante para el avance del pensamiento crítico, difundido a través de lo que podría ser la educación soportada en una pedagogía alternativa, cuando no edificadora; en este caso, desde las discursividades de los docentes ocupados de enseñar televisión, $y$, de paso, las nuevas formas de advertir contenidos a través de otros dispositivos y multipantallas.

\subsection{Nuevas formas de ser y estar frente a la pantalla}

\subsubsection{La Remediación y el Binge-watching}

Los nuevas formas de ser y estar frente a la pantalla para el consumo audiovisual, han puesto en evidencia la disrupción de modelos teóricos de análisis y actitudes del hombre en su interacción social (Halliday, 1979); Justamente, frente a la invención televisiva que ha sido permanente en su función desde su creación hasta el presente siglo; no obstante, no en sus formas ni modalidades.

Según Hernández y Martínez (2017), dicha disrupción tecnológica que supuso la digitalización de la señal audiovisual, ha convertido a los consumidores de productos audiovisuales en usuarios activos debido a la participación a la que tienen lugar y al cambio de paradigma técnico que ha favorecido la aparición de nuevas formas de acceso. Según sus investigaciones, estos hechos han propiciado la modificación inevitable de la relación entre el público y los contenidos. Para ellos, el origen de este trasunto, estriba en el uso masivo a través de Internet y de múltiples instrumentos que han posibilitado a los internautas, el libre intercambio de cualquier material fílmico (videos, películas, series, novelas, audios, etc), alejados de algún tipo de restricción comercial o configuración modelada por la producción y edición tradicional.

Por tales razones, afirman los investigadores, se ha incrementado exponencialmente tanto los títulos de consulta disponibles para un consumo inmediato, como las posibilidades de accesibilidad y participación activa a un coste muy bajo o a cero costes. Esto es lo que se define hoy como el fenómeno bingewatchin (Maratón televisivo), (David Bolter y Richard Grusin, 2011), que en gran parte de los consumidores del globo ha modificado su conducta. Lo que tiene incidencia directa en los medios de comunicación tradicionales y en los hábitos de comportamiento del espectador en la relación: pantalla-vida cotidiana.

Partiendo de las ideas de Bolter y Grusin (2011:51), Hernández y Martínez asumen que la omnipresencia de Internet contribuye con un gran poder de decisión, para que el internauta, quien ha mutado de forma definitiva sus hábitos de consumo, decida cómo, cuándo y dónde consumir cualquier contenido audiovisual. Una acción que, según su mirada, ha experimentado un crecimiento en la mayor parte de la audiencia actual; entre estos, los jóvenes universitarios.

Lo anterior, proporciona la economía de la abundancia en contraposición de modelos clásicos de la escasez. Esta dinámica lo que le posibilita al espectador-internauta es el descubrimiento continuo de productos nuevos, 
a la vez sugeridos por otros usuarios a través de múltiples plataformas; cuyas interacciones configuran redes virtuales de información y comunicación sincrónica y asincrónica.

De ahí, que para los investigadores cobre relevancia lo planteado por Bolter y Grusin cuando indican que la televisión debe ir amoldándose a las necesidades de un nuevo perfil de receptor que se hace en paralelo a la evolución tecnológica. Lo que es denominado como "proceso continuo de remediación, esto es, la representación de un medio en otro medio" (2011:51). Lo que conduce con seguridad a una competición entre televisión y red que, según ellos, parece no tener un punto de vista común.

Esta nueva categoría supone entonces la posibilidad de volver a mediar o si se quiere, auto-generar un proceso de reproducción de contenidos de una pantalla a otra, seleccionando, controlando y avanzando en la observación a ritmos propios, en una suerte de visionado de capítulos consecutivos; sobre todo, aquellos basados en series de ficción.

Esta nueva actitud de sentido frente a la pantalla, es lo que, de acuerdo con Hernández y Martínez (2017), genera afectaciones en el usuario ante las diversas "estrategias que tiene a su disposición para apostar por un consumo mediático a la carta, gestionado por él mismo" (p. 3). De este modo, se mantiene en este escrito, que al parecer ha quedado relegada en gran medida la programación estándar televisiva y ha aparecido una nueva situación colmada de opciones ajustables a las necesidades de cada usuario.

Por tanto, el papel de la academia, en este sentido, tendría que transitar a nuevos circuitos e interacciones pedagógicas, para no advertirse como una institución con prácticas obsoletas; por ejemplo, confinada a una tradición pedagógica logocéntrica o inquisitiva; aún más, cuando el avance de la tecnología, los descubrimientos y relaciones novedosas en las ciencias formales (lógicas-ideales) y fácticas (empíricasmateriales) que tenemos a nuestra disposición, siguen ofreciendo sorpresivos adelantos que reevalúan conceptos y aplicaciones pasadas.

Continuar con una academia anquilosada, para el tema que nos ocupa, en específico, significaría un obstáculo para determinar si efectivamente los hábitos frente a las pantallas generan discursividades y aprendizajes nuevos, o por el contrario, lo que se ha configurado es un mito a través de las nuevas construcciones sociales de la realidad, frente a las formas nuevas de ser y estar frente a la televisión, las multipantallas y los contenidos de diversa índole. En lo global, significaría un mayor estancamiento en la gestión educativa latinoamericana, cuyas cifras en el ámbito educativo internacional, no favorecen ni la inversión, ni el desarrollo social y cultural de la nación, que permitan cerrar brechas en los niveles de pobreza y formación humana.

Es preciso entender, en este sentido, que actualmente los modos de ver, ser, estar y sentir frente a las pantallas se han vuelto subjetivos, personales e íntimos, en relación con lo que en el pasado significaba estar frente a ese mundo llamado: televisión. De alguna manera, la colectivización de la pantalla ha dado paso al enclaustramiento del sujeto en una burbuja subjetiva, la cual se abre y se cierra al antojo del internauta, cuando este lo desea; ya sea a través de la socialización por redes, o por medio del propio discurso en los momentos de interacción física; normalmente, en ámbitos sociales como las instituciones, la calle o el entorno familiar.

En este contexto se plantea lo que significan las innovaciones disruptivas. Que, relacionadas con lo anterior, no son más que las nuevas maneras de proceder del usuario quien se encarga con sus hábitos de visionado de las "modificaciones que reestructuran la esencia de un elemento concreto" (Hernández y Martínez, 
2017: 3). Hacen parte, no obstante, de lo que estos autores dan en llamar: "evolución en el consumo de productos audiovisuales". Parece ser que en el ámbito de la industria, las mutaciones o modificaciones realizadas por el usuario, adicionan mejoras significativas a los contenidos, desplazando progresivamente los productos y servicios ofrecidos por empresas reconocidas como líderes en el campo.

Por consiguiente, la innovación disruptiva propuesta por los investigadores y en sintonía con lo fundamentado por Bolter y Grusin (2011), en referencia al vinge-Watching, y la re-mediación, se torna para los nuevos estudios comunicacionales, elementos de obligatoria inclusión en modelos teóricos y analíticos contemporáneos, preocupados por estudiar las nuevas formas de ser y estar de los usuarios frente a las pantallas; sobre todo, de las comunidades estudiantiles y juveniles, como es tratado aquí, quienes con ahínco han venido mostrando transgresiones, transmutaciones, reconfiguraciones, reconstrucciones y transcreaciones, objeto de análisis y comprensión para el nuevo orden mundial y tecnológico que se teje.

En esta medida, entender la innovación disruptiva o lo que para el presente estudio se estima como las alteridades y alternativas juveniles contemporáneas, supone comprender cómo las nuevas tecnologías de la comunicación han desplazado las bases de modelos vejetes del negocio de la industria audiovisual, que requiere de la producción, distribución y promoción de contenidos, para luego entender las nuevas formas de ser y estar del usuario frente a las pantallas.

Constituye un hecho, entonces, que las nuevas maneras de acceder a los contenidos a través de múltiples plataformas, redes, servicios y dispositivos, etc., posibilitan una gran variedad de beneficios, decisiones, materiales y accesibilidad interactiva sin fin. Esta diferencia frente a las posibilidades de antaño, hacen que, como lo refieren los autores, las conductas y hábitos cambien, pero también las discursividades y maneras de denominar.

De ahí que, el lenguaje en este horizonte, sea determinante en la medida que crece, muta, avanza o muere, en concordancia con la progresión tecnológica y los nuevos modos de significación. Aunque este aspecto no es resaltado en las investigaciones de los autores referidos aquí, es para la presente indagación un aspecto fundamental, indudablemente hallado en el centro de toda construcción humana; pues es un hecho que las prácticas discursivas en torno a los prolíficos crecimientos tecnológicos, van dando cuenta de las lógicas de sentido en que las sociedades van interactuando y concertando sus modos de ser, hacer y sentir.

\subsubsection{Cant stop - Wont stop Binge-Viewing}

Relacionado con lo referido anteriormente, el título de este ítem da cuenta de una investigación realizada en el año 2014 por Ciaramella y Biscuiti, en la que se alerta sobre el fenómeno binge-watching, como una de las dinámicas contemporáneas de mayor impacto en el usuario de la pantalla moderna. El estudio denominado: “Can't Stop, Won't Stop: Binge-Viewing Is our New Favorite Addiction (2014)", concluye que siete de cada diez norteamericanos se asumen como espectadores de nivel binge-watching.

Se trata así de un análisis conductual de la audiencia llevado a cabo a través de 800

8 Con esta composición nos referimos a las formas disruptivas de los jóvenes contemporáneos, para afrontar las construcciones sociales de la realidad cotidiana en sus dinámicas de hiperconectividad. Esto es, las acciones poco convencionales y fundamentalmente transversalizadas por los dispositivos, plataformas, redes y multipantallas tecnológicas, para explicarse su sentir, entender el mundo e interactuar con él; acciones que no son tan comprendidas ni aceptadas por algunos sujetos, que, aunque experimentando el actual orden socio-tecnológico, siguen siendo renuentes a las re-creaciones progresivas o, en su defecto, permanecen cosificados en lo tradicional, cuando no signados en tiempos y relaciones educativas, sociales, culturales y tecnológicas pasadas. 
encuestas en las que se determinaron los comportamientos de los propios encuestados, en relación con la modificación del consumo audiovisual, tal como se ha dicho (2017:4-5). Lo que en el contexto colombiano, y en específico con los estudiantes de la Licenciatura en Comunicación e informática educativas de la Universidad Tecnológica de Pereira, se desea constatar o refutar.

No poder parar o no querer parar en el maratón televisivo, constituye una nueva modalidad de ver contenidos audiovisuales. Para el caso aludido, el estudio permitió concluir sobre los diferentes comportamientos que se alteraban por dedicar un tiempo excesivo al visionado de cualquier tipo de contenido; en especial, se determinó sobre el número de capítulos visionados sobre series de ficción.

No distinto a lo que acontece en América Latina, la influencia de diferentes portales, plataformas y dispositivos, hace que el cant stop-wont stop se difumine a través de las sociedades y sus nuevas prácticas de consumo y discurso a través de entornos virtuales y accesos asincrónicos y sincrónicos, generadores de infinitud de opciones receptivas y comunicativas.

Es un hecho que los jóvenes en edades escolares de secundaria y universitaria, no solo recurren a tales prácticas, sino que, al parecer, se ha convertido en una suerte de competencia entre ellos para determinar quién o quiénes han visto primero un material de interés sociojuvenil y quiénes después. Parece ser, también, que quienes no se han acercado a los títulos, capítulos y series más prolíficas, cuando no populares, caen en la crítica y discursividades del otro. Sobre todo, a través de redes sociales donde se comenta con desmesurado afán, inagotable difusión y cruda referenciación.

De esta manera, según Longwell, 2014, citado por Hernández (2017), el estudio reseñado concluyó que los comportamientos modificados en favor del consumo audiovisual, son los relacionados a continuación:

- Higiene cuestionable: de forma habitual, aquellos espectadores que se consideran binge-viewersson más proclives a aplazar una ducha o un baño por ver su serie favorita.

- Menor actividad: un porcentaje elevado de los encuestados afirman sentirse, en términos generales, lentos y perezosos cuando realizan un consumo excesivo.

\section{- Prestan menos atención a otros} aspectos de su vida: de forma frecuente los binge-viewers modifican sus hábitos alimenticios a través de un mayor consumo de platos a domicilio u olvidando una comida completa. Del mismo modo, alteran su rutina de sueño en favor del visionado compulsivo.

En general, estas modificaciones ocasionadas por la fusión entre pantalla-contenidos-vida cotidiana, revela el desenfrenado interés del usuario contemporáneo, por los contenidos de alto impacto e interés audiovisual, como los seriados y las propuestas de carácter novedoso, cuando no transgresor; cuyos contenidos, son dirigidos a las subjetividades de los individuos, como si constituyeran una especie de targets identificados para ser seducidos por la dinámica propia de las grandes plataformas, fundadas en la libertad para observar, intervenir, comentar, reproducir, re-transmitir, prescribir o simplemente, diluir. Parece ser que la actual sociedad privilegia estos modos de ser y estar frente a las pantallas, como una suerte de dimensión naturalizada en los espectadores de la acelerada centuria.

\subsubsection{Dimensiones de un nuevo espectador según Ferguson}

Hacer la diferencia entre el espectador neutral del pasado con el espectador activo del presente, Hernández y Martínez (2017), 
implica referir a Ferguson, 2014, como uno de los teóricos que ha llamado la atención sobre las nuevas conceptualizaciones del usuario activo frente a las pantallas, contenidos y producciones ofrecidas actualmente. Dicho autor, esboza categorías como: creadores, remezcladores, compartidores y prescriptores, que atendiendo a lo ya planteado desde posturas socio-culturales y pedagógicas pasadas (Maturana 1992; Canclini, 1999), entre otros), pero de gran vigencia para la sociedad contemporánea, apuntan a una re-conceptualización de ideas y de acciones en el ámbito del consumo y la comunicación audiovisual. Se trata de nuevos lenguajeares a la visión de Maturana, que permiten, como en este caso, entender no solamente el avance nominativo, sino lo que se halla de fondo en la relación: leguaje- conocimiento-acción.

Así, con Ferguson, 2014, se entiende que la irrupción de la tecnología, las nuevas experiencias en los entornos virtuales del usuario y las posibilidades de intervención en los contenidos, ha impulsado mejoras técnicas promovidas por la digitalización y ha convertido a los múltiples usuarios mundiales en coparticipantes, cumpliendo roles inter-activos e inter-discursivos en el escenario de la vida virtual y su recepción. Según Ferreiro, "ahora somos mucho más libres que antes en nuestra relación con los contenidos" (2012:1).

Sin duda lo somos. Y como se ha expresado, los hábitos y tendencias de consumo audiovisual en la población joven, se han transfigurado ostensiblemente. Así, en sintonía con Hernández y Martínez (2017), y otras investigaciones (López Vidales, 2012), la población de jóvenes con edades comprendidas entre los 14 y 25 años es la que menos consume contenidos audiovisuales directamente de la televisión tradicional, pues prefieren acceder por medio de dispositivos digitales y móviles. Esto contribuye, como los investigadores precisan, a que se haya sustituido a gran escala la televisión como vía para el ocio juvenil, siendo reemplazada por la diversidad de acciones posibilitadas por la Red, en lo general, y por adición a la ficción seriada, en particular. Los estudios citados por Hernández y Martínez (2017:4), así lo demuestran. Al respecto los autores refieren lo planteado por Chicharro:

[...] el ordenador personal se ha convertido en la plataforma preferida por los universitarios para el visionado de este tipo de contenidos audiovisuales, muy por encima de la televisión o el DVD. Las razones principales para esta elección se centran en la posibilidad del consumo individual y repetido del material elegido, sin la necesidad de litigar por el producto a ver. En este sentido, «los productos de ficción pueden así ser consumidos cuando se quiera, en la dosis, o «sobredosis» deseada, sorteando las pausas publicitarias, e introduciendo cortes o descansos en el visionado, ganando autonomía, en definitiva, sobre las cadenas emisoras» (Chicharro, 2014: 84).

De este modo, la innovación disruptiva y estos nuevos modelos de consumo generados por los propios usuarios, y beneficiados por las mejoras tecnológicas relacionadas con Internet, así como por el uso del intercambio no comercial de contenidos, ha impulsado las siguientes transformaciones en el consumo de productos audiovisuales, según Grandío, 2011, citado por Hernández y Martínez (2017:5):

- Ruptura de intermediarios geográficos y temporales en la difusión y acceso a cualquier contenido. Así, un espectador puede visionar un título concreto desde cualquier lugar del mundo pocas horas tras su estreno cinematográfico o televisivo.

- Laglobalización en la distribución audiovisual 
provoca una especial predilección por las ficciones en habla inglesa.

- El público más joven resulta el mayor consumidor de aquellas herramientas que permiten el acceso no comercial a productos fílmicos.

- El consumo de material cinematográfico o televisivo en grupo ha disminuido en pos de un deleite individual de ocio promovido por plataformas informáticas unipersonales como el ordenador personal, el teléfono móvil o la tableta táctil.

- La globalización en la distribución de productos audiovisuales que provoca el intercambio no comercial de contenidos ha elevado el consumo de productos en versión original. En consecuencia, y ante la imposibilidad de la industria del doblaje de seguir el ritmo de visionado de este nuevo público, han surgido multitud de sitios web especializados en labores de subtitulado, la gran mayoría de ellas sin ánimo de lucro.

- La enorme cantidad de material cinematográfico y televisivo de libre de acceso que inunda Internet produce en el espectador un incremento en el consumo televisivo. Este mecanismo, que se hace muy evidente en los contenidos seriados de ficción, provoca que una gran cantidad de espectadores visionen varias series de televisión al mismo tiempo, en consonancia con su emisión televisiva original. A su vez, este tipo de consumidores, son capaces de visionar varios capítulos consecutivos en un proceso de atracón conocido como bingewatching.

Este último comportamiento es definido como adictivo si el ritmo de visionado supera los tres capítulos consecutivos. Un hecho que en muchos casos se reitera y toma fuerza en la población juvenil escolar secundaria y universitaria. De acuerdo con Hernández y Martínez (2017), "un estudio de la consultora estadounidense Miner \& Co. Studio aclara que el espectador con binge-watching es capaz de modificar sus hábitos de comportamiento en pos de consumir su contenido audiovisual preferido (Ciaramella y Biscuiti, 2014)".

Cambios que están relacionados al tiempo con lo planteado arriba por Ferguson en cuanto los sujetos de la actual centuria como usuarios: creadores, remezcladores, compartidores y prescriptores. En su orden, entendemos estas categorías como las dimensiones de un nuevo espectador así:

Creadores: Sujetos-usuarios de la contemporaneidad digital, capaces no solo de consumir diferentes clases de contenidos audiovisuales a través de diferentes plataformas y dispositivos, sino también, aquellos que son capaces de crear sus propias producciones a través de posibilidades y herramientas dadas a los internautas sin coste, en algunos casos, a través de las cuales dicen sobre su mundo, intereses, perspectivas, proyecciones y preocupaciones en el desarrollo individualcolectivo en el que se halla inmerso tanto física como virtualmente.

Remezcladores: Dadas las posibilidades que le ofrece internet a los usuarios, entendemos con Ferguson (2014), que el usuario re-mezclador es aquel que vuelve a mezclar los contenidos audiovisuales una y otra vez en un proceso de transcreación y recreación de sus experiencias iniciales. Esto es, la posibilidad actual de generar nuevas narrativas a partir de las ya creadas o tradicionalmente reseñadas por la oralidad, la literatura, la historia y los medios masivos clásicos.

Compartidores: En acuerdo con la teoría de Ferguson (2014), se entienden como los usuarios que a través de sus multi-dispositivos y plataformas, se permiten compartir diversidad 
de contenidos audiovisuales, desde fotografías, videos, producciones hechas, propias, recreadas, comentadas, etc, para dar respuesta a la necesidad individual y colectiva gestada en las nuevas formas de ser y estar frente a las pantallas.

Estas decisiones de compartir lo que se quiera sin restricción, da pie a la configuración de la individualidad y la generación de identidades propias de la modernización y sus desarrollos eclécticos, híbridos, cuando no simultáneos. Compartir, ser colectivo en la diferencia en este siglo, parece ser uno de los rasgos más característicos en progresión del mundo modernizado, a pesar de individualidades, sesgos y visiones retrógradas en gran parte de las áreas del conocimiento humano. El papel de la academia, en esta medida, estaría llamado a ser pluralista en su fondo pedagógico y en su función educativa, política y social.

Prescriptores: Son entendidos a la luz de lo planteado por Ferguson (2014), como aquellos usuarios-internautas capaces de influir en un determinado público con sus opiniones, valoraciones y/o decisiones. En este caso, sobre contenidos audiovisuales comentados, reseñados a través de audios, escritos y videos que pueden hacerse virales y tener efectos de persuasión masiva. Los de mayor alcance serían aquellos dotados de un lenguaje que refleje poder y autoridad.

Así, cualquier usuario dotado de estos atributos, como el caso de algunos Youtubers, periodistas, políticos o personalidades públicas, pueden convencer de la posición positiva o en contra relacionada con alguna temática de público conocimiento e interés social, político, educativo, deportivo o de sencilla farándula. Aunque en ello, también, pueden gestarse los casos de las New Fake, tan posibles desde la re-creatividad virtual, como difundidas hoy en día a través de cualquier plataforma y dispositivo.
Es por tanto, que el estudio del que se deriva este acápite, se concentra en identificar, categorizar y analizar las prácticas de los estudiantes de la Lic. En Comunicación e Informática Educativas frente a la pantalla televisiva, en momentos significativos de su avance curricular en la carrera, que al enmarcarse en las nuevas formas de ser y estar frente a las pantallas, le sirvan de insumo académico y diagnóstico al docente de la asignatura Televisión, para apoyar sus discursividades dentro del aula, al tiempo que potenciar nuevas formas de acercarse a la construcción social de sus estudiantes en relación con los visionados, la televisión, las multipantallas y sus particulares miradas sobre el mundo; las cuales, probablemente, comprometen sensibilidades nuevas frente a su propia educación, creación y conocimiento geomundial, cuyas sinergias, en otros tiempos, no eran de tan acelerada detonación tecnológica y virtual.

\section{EL PAPEL DE LA ACADEMIA EN LA TENSIÓN ENTRE LAS VIEJAS Y LAS NUEVAS FORMAS DE SER Y ESTAR FRENTE A LAS PANTALLAS}

Con este panorama teórico se asume que el papel de la academia en dicha tensión, podría estar comprometida con una pedagogía alternativa, progresiva y edificadora. Esto significa en el marco de prácticas pedagógicas aún tradicionales, una mirada diversa e inclusiva para determinar si hay nuevas formas de ser y estar frente a las pantallas, o no ha habido variaciones esenciales en contraste con las viejas formas, a pesar del despunte tecnológico.

No obstante, pensar en un papel de la academia desde un punto de vista alternativo y edificador, tendrá que ver con realizaciones y experiencias signadas en el análisis, la interpretación y la corroboración de datos surgentes en los propios desarrollos y posturas de los estudiantes en relación con sus procesos de formación disciplinares; en este caso, como se 
ha mostrado, el papel de la academia podría estar en la exploración de la televisión y las multipantallas en el aula, como espacios de construcción de saberes para el desarrollo social, más que individual. En esta idea, toma lugar la que Rincón (1995) ha sugerido desde una postura crítica en el ámbito de las prácticas educativas:

Para comprender cómo la televisión hace de la educación una acción espectacular y formadora, debemos transformar nuestra concepción de este medio audiovisual. Este desplazamiento consiste en pasar de la concepción de televisión como medio de información, recreación y educación, a una comprensión de la misma como campo de producción de saber social. La televisión es, entonces, el lugar donde se legitiman ciertas maneras de percibir y representar, de premiar y castigar, de valorar y denigrar. La televisión es una manera de participar, un lugar de encuentro y un ámbito de construcción de la realidad (Omar Rincón, 1995).

Esta referencia no es más que pertinente con el papel que la academia podría explorar alrededor de las pantallas, pensando en la consolidación sería del saber para la obtención de un hombre formado política y éticamente, en función de una mejor sociedad.

Es un sentir que se correlaciona con otra idea del autor, según la cual, la televisión no puede clasificarse por los contenidos culturales o no culturales, educativos o no -educativos que transmite, sino por las formas de significación y las sensibilidades que propone. Esto significa, en su contemplación, que la función cultural y educativa de la televisión no pasa por los contenidos, sino por las formas de comunicar, por las voces que representa, por las sensibilidades que expresa, por los estilos de vida que proponer, por los modelos de justicia que socializa, etc.
Para el autor, sería interesante que la televisión comenzara a funcionar como constructora de redes de sentido para una sociedad (Rincón, 1995).

Estas consideraciones sobre la televisión, pone en un lugar de altísimo cuidado el papel pedagógico del docente en los estilos de enseñanza. Como lo hemos dicho, entonces, y asumiendo la voz de rincón, el sentido de la educación no podría destacarse exclusivamente por los contenidos, como muchas de las visiones pedagógicas de avanzada hoy lo proponen (Maturana, 1992; 1999; 2002; Mc Laren, 2003; Giroux, 1992; 1993;1996;1997). Las relaciones deberían tejerse en la interacción entre los sujetos, sus sentires y pluralidades, las cuales permitan reavivar el sentido dialógico y democrático de la nación, y todo lo que en ella circula.

En suma, a continuación referimos las exploraciones-acciones que la academia, cumpliendo un papel alternativo y edificador, podría desarrollar de cara a un continuum abierto, plural y detonante en el contexto de la educación latinoamericana y la tensión entre las viejas y las nuevas formas de ser frente a las pantallas:

1) Reconocer las viejas formas de ser y estar frente a las pantallas, para explicar las bondades y limitantes de las nuevas, de cara al crecimiento del ámbito académico y socio-cultural. Valorar los aportes del pasado es la acción más ética de la academia para criticar, argumentar y proponer nuevos rumbos científicos.

2) Incorporar efectiva y atractivamente la televisión y su análisis en el aula de clase, teniendo en cuenta las relaciones entre contenidos, prácticas sociales, otras pantallas, contextos e intereses de los estudiantes universitarios; ciertamente, de aquellos matriculados en una carrera 
profesional en la que la televisión constituya una asignatura de estricto cumplimiento en el programa.

3) Analizar y socializar en el aula de clases, las construcciones sociales de la realidad que los estudiantes realizan a través de sus discursividades, en relación con el mundo, sus intereses, sentires, proyecciones, creaciones; esto es, comprender la psicología individual y colectiva de los jóvenes en su interacción con la televisión y las multipantallas, para revisar comportamientos, hábitos, en provecho de las discursividades docentes y una atmosfera educativa inclusiva, circular, pluralista, alejada de hegemonías y linealidades pretéritas.

En la siguiente figura se puede visualizar lo dicho:

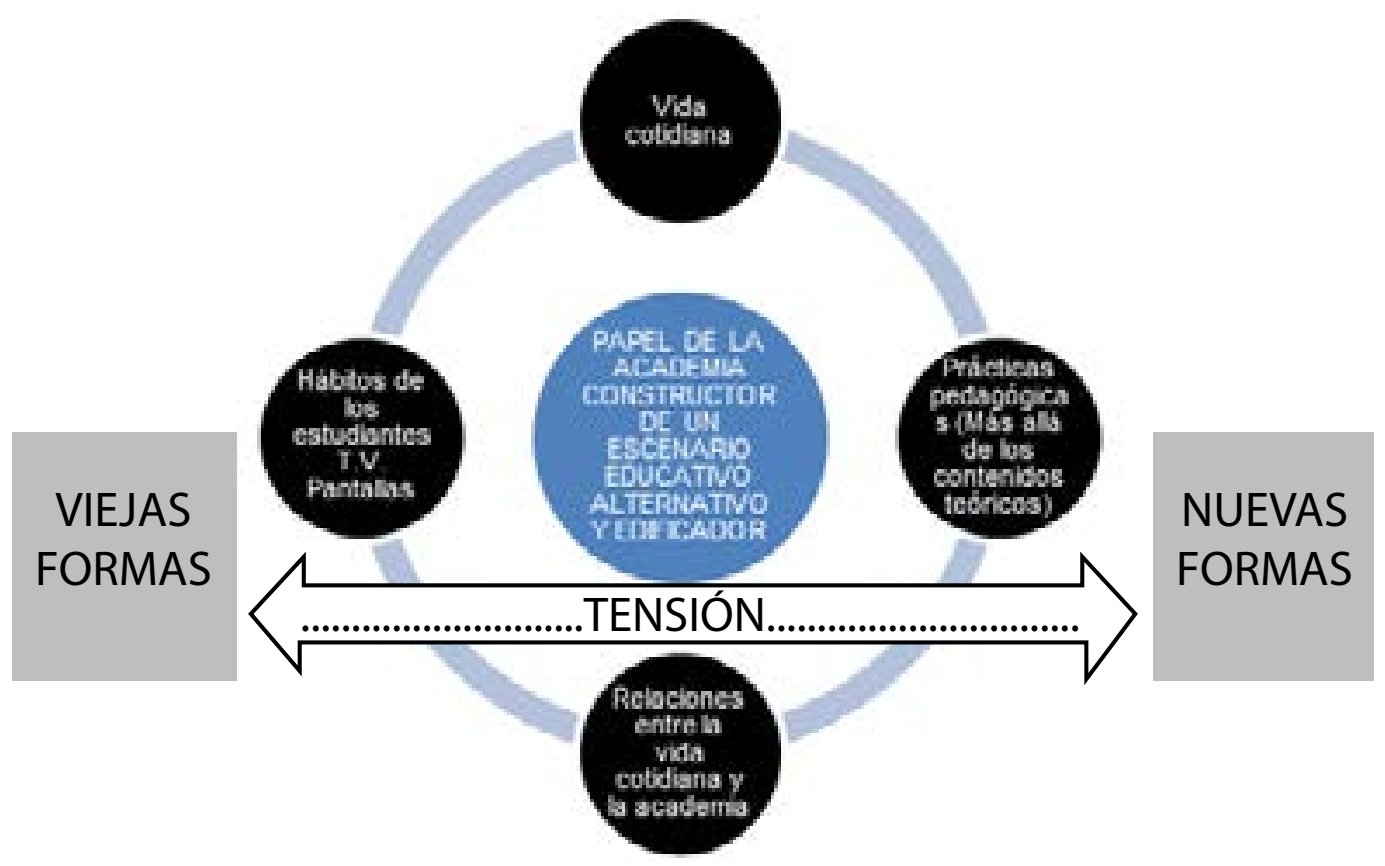

Fig. 1. El papel de la academia en la tensión entre las viejas y las nuevas formas de ser $y$ estar frente a las pantallas

4) Explorar características pedagógicas fundamentadas en dinamismos y variaciones en las formas de didactizar el conocimiento referido a la televisión, las multipantallas y sus implicaciones tecnológicas, para comprender las nuevas formas de ser y estar frente a las pantallas de los estudiantes universitarios, basadas en las relaciones entre saber-placer /vida-contenidos; de este modo, las renovaciones didácticas, curriculares y evaluativas, tendrían lugar como hechos móviles en el devenir natural de la vida y sus transformaciones.
5) Pensar en la función social de las prácticas y modificaciones ocasionadas por la fusión entre pantallas, contenidos y vida cotidiana. Esto es, diseñar rutas de trabajo entre estudiantes y docentes, que permitan impactar los contextos familiares y socioculturales en los que vive cada estudiante. Significaría, entonces, realizar proyectos de aula activos y relacionados con la televisión y las multipantallas, de suerte que incorporen la familia y la comunidad. 
6) Las posibilidades de intervención en los contenidos de T.V. y las técnicas promovidas por la digitalización deben catapultar al estudiante hacia roles interactivos, interdiscursivos y co-participantes, más allá de una educación tradicional y xenófoba, relativa a los medios y las pantallas; sobre todo, de la televisión y sus posibilidades actuales de re-mediación.

7) El papel de la academia en sus exploraciones pedagógicas, debería estar signado en clave freireana y nietszcheana. Así la perspectiva libertaria y detonante en la tensión entre las viejas y las nuevas formas de ser y estar frente a las pantallas, transitaría caminos socio-democráticos entre el logos (razón) y la sensibilidad (emoción) del estudiante universitario hiper-conectado.

8) Atender a las circunstancias y situaciones contextuales en los diferentes ámbitos educativos, en el marco de la hiperconectividad global, las pedagogías progresistas y las discursividades alternativas y edificadoras en el aula.

9) El papel de la academia en esta tensión descrita, en conclusión, podría atender (en sus exploraciones y didácticas logradas a partir de los insumos discursivos de los estudiantes) las circunstancias y situaciones dadas en los diferentes contextos educativos, en los que la televisión sea enseñada como parte de un programa académico universitario. Naturalmente, no apartada del marco tecnológico y de hiperconectividad global, ni de las pedagogías progresistas que edifican y dignifican el aula y el hombre en la sociedad. Son pues exploraciones pedagógicas y didácticas que, en el universo del papel de la academia en la tensión abordada, podrían detonar, soportada en la libertad para re-pensar el mundo y sus posibilidades futuras, su Kairós.

\section{CONCLUSIONES PREVIAS}

Una perspectiva como la presentada, posibilita no solo la recreación de los fundamentos comunicativos y discursivos para un insumo que permita las discursividades y prácticas educativas del docente en el aula, sino también la proyección aplicativa en función de la sociedad y su cualificación educativa, ética, estética y democrática.

La presente reflexión en el marco de la investigación de la que procede, puede lograr que los estudiantes potencien su mirada hermenéutica, reflexiva y crítica, frente a los contenidos ofrecidos por la televisión colombiana en formatos tales como: Noticieros, Reality Shows, novelas, seriados, películas, etc.

La mirada del estudiante universitario que se enfrente a procesos de enseñanzaaprendizaje relacionados con la televisión, las pantallas y la tecnología en general, puede ser afinada en sintonía con producciones discursivas críticas, atentas del diálogo entre el fundamento teórico y las posturas propias que emergen en el proceso de formación.

Esta posibilidad, les permite a los estudiantes plantear y adaptar dinámicas similares en el aula de clases en el momento de su actuación pedagógica. Lo que indica que serán docentes replicadores, analíticos, cuando no propositivos en relación con las formas de ver y estudiar la televisión en los niveles de educación en los que ejerzan. En este sentido, serán conscientes de que existen mediaciones entre la pantalla, la audiencia y la participación en la televisión colombiana, que precisa ser enseñada a partir de posturas críticas y pedagógicas edificadoras. 
- Como observatorio y estrategia de comunicación en el aula universitaria, la propuesta contribuye al desarrollo de un pensamiento autónomo, líder y propositivo en el ámbito educativo colombiano.

- Favorece a la formación de audiencia en el estudiante universitario de la Licenciatura en Comunicación e Informática educativas, para contribuir a la formación de otros en el ámbito familiar, social y educativo en el que co-participe.

- Los fundamentos teóricos nos permiten pensar y analizar modos diversos de ser y estar frente a la pantalla chica y otras pantallas, en los que la interacción activa y los nuevos lenguajeares, dan cuenta de nuevos hombres, pensamientos y acciones en la sociedad.

- El desarrollo del trabajo investigativo se constituye en un observatorio, y, a la vez, repositorio o centro de documentación, en el que anualmente puede generarse informes sobre la percepción de los estudiantes del programa, objeto de estudio, en relación con la televisión, las multipantallas y los formatos ofrecidos continuamente en varias plataformas tradicionales $y$ contemporáneas.

- En tanto se trata de un trabajo en continuo monitoreo y aplicación, su dinámica sería posible replicarla y ampliarla en otros contextos educativos de Colombia, para obtener datos comparativos que permitan hacer una radiografía de nuestra sociedad y sus formas de asumir y ver la televisión, en relación con la población juvenil universitaria pertinente; naturalmente, que en el contexto de las transformaciones propias de la vida, las cosas, las situaciones específicas, los avances educativos y tecnológicos, en los que ubicamos el fenómeno en cuestión. Pues como ha sido precisado en el fondo histórico, pedagógico y filosófico que nos asiste, la vida, el hombre y sus interacciones no pueden estar más que destinadas a la metamorfosis, donde las tensiones se convierten en elementos perdurables, pero al tiempo dialécticos y variables en el existir humano. Esto significa para una pedagogía edificadora y alternativa, comprender que la acelerada era tecnológica en la que nos hallamos, es el reflejo de un logos en movimiento, contrastivo y enérgico; más allá, como ya ha sido demostrado por la historia, de la rigidez metafísica y su tradición (...)

\section{REFERENCIAS BIBLIOGRÁFICAS}

- Bernal, César A. (2010). Metodología de la investigación. Administración, economía, humanidades y ciencias sociales. $3^{\text {a }}$ edición. Colombia, Pearson Educación, de Colombia Ltda.

- Bustamante, Boris y Aranguren, Fernando (2012). Colombia, Del unanimismo discursivo a la apertura, la innovación y la internacionalización. Obitel. 2012.

- Castells, Manuel (2010). Comunicación y poder. Madrid, Alianza Editorial. Capítulos I-III. Pp. 21-259.

- Krippendorff, K. (1990). Metodología de análisis de contenido. Teoría y práctica. Barcelona, Paidos Comunicación.

-Deslauriers, Jean- Pierre (2005). Investigación cualitativa. Guía práctica. Colombia-Pereira, editorial Papiro.

-Freire, Paulo (1970). Pedagogía del oprimido. Nueva York, Herder y Herder. (Manuscrito en portugués 1968) Publicado con el prefacio de Ernani Maria Fiori. Río de Janeiro, Continuum, 218 p.) 
- Luhmann, Niklas (1990). Teoría de la sociedad. Guadalajara, México, Universidad Iberoamericana, 1993.

- Martín- Barbero, Jesús \& J. Y muñoz, S. (Coords.) (1992). Televisión y melodrama. Géneros y lecturas de la telenovela en Colombia. Bogotá, Tercer Mundo Ediciones; pp. 19-60.

- Martín-Barbero, Jesús (1987). De los medios a las mediaciones. Comunicación, cultura y hegemonía. Barcelona, Gustavo Gili; pp. 31-43 y 229-241.

- Martínez Solís, María Cristina (2001). Análisis del discurso y práctica pedagógica. Tercera edición. Argentina, Editorial Homo Sapiens.

-Maturana, Humberto (1999). Transformación en la convivencia. Santiago de chile, editorial universitaria.

-Maturana, Humberto (2002). Emociones y lenguaje en educación y política. Santiago de chile, dolmen ediciones.

-Maturana, Humberto (1992). La objetividad. Un argumento para obligar. España, Domen ediciones.

-Mc Laren, Peter (2003). La escuela como un performance ritual. Hacia una economía política de los símbolos y gestos educativos. Tr. Sebastián Figueroa Rodríguez. México, Siglo XXI. S.a de c.v. Centro de estudios de la Universidad Unam.

•Morley, D. (1996). Televisión, Audiencias y Estudios Culturales. Buenos Aires, Amorrortu editores.

- Neumann, N (1992). El nuevo espacio público. Barcelona, Gedisa; Capítulos 20 y 21.

- Orozco, G. y R González (2012). Una coartada metodológica. Abordajes cualitativos en la investigación en comunicación, medios y audiencias. México, Tintable.
- Orozco, G. y R González. Desafíos educativos en tiempos de "auto-comunicación masiva". Una propuesta para la interlocución de "viejas y nuevas" audiencias. México.

- Orozco, Guillermo (2001). Televisión, audiencias y educación. Buenos Aires, Norma: pp. 11-113.

- Orozco, Guillermo (2012). La investigación de las audiencias "viejas y nuevas". Versión actualizada de la conferencia inaugural del congreso nacional anual (2010) de la AMIC: Asociación Mexicana de investigadores de la Comunicación.

-Rey, Germán (2002). "La televisión en Colombia", en OROZCO, Guillermo (Coordinador), Historias de la televisión en América Latina, Barcelona, Editorial Gedisa.

- Wolf, M. (1987). "Contextos y paradigmas en la investigación sobre los media" en Wolf, M. (1987). La investigación de la comunicación de masas. Crítica y perspectivas. Barcelona, Paidós.

- Wolf, M. (1987). La investigación de la comunicación de masas. Crítica y perspectivas. Barcelona, Paidós.

- Zuleta, Estanislao (1985). La educación: un campo de combate. Entrevista a Estanislao Zuleta. Revista Educación y Cultura \#4, FECODE. Bogotá. 1985.

\section{WEBGRAFÍA}

-AlonsoA. María. M(2011). Televisión, Audiencias y estudios culturales: Reconceptualización de las audiencias mediáticas, En: "Razón y Palabra". Primera Revista Electrónica en América Latina Especializada en Comunicación. Libros Básicos en la Historia del Campo Iberoamericano de Estudios en Comunicación. Número 75 FebreroAbril 2011. www.razonypalabra.org.mx 
-Arboleda, J. C. (2016). Formación para la vida: de las competencias a la comprensión edificadora. Revista Boletín Redipe, Vol. 4, №. 12, 2015, págs. 20-25.

-Arboleda, J.C. (2018). La educación es inclusiva: Perspectiva comprensivo edificadora de la formación. En: "Tarea crítica de la Educación: contingencias epistémicas, emergencias metodológicas y discusiones para el presente. Ciclo de Conferencias internacionales organizado por Celei: https:// www.celei.cl/la educacion-inclusiva-desdela-perspectiva-comprensivo-edificadorade- la formacion-julio-cesar-arboleda/

-Ciaramella, D., \& Biscuiti, M. (2014). Can't stop, won't stop: binge-viewing is our new favorite addiction. Nueva York: Miner \& Co. Studio.

-Chicharro Merayo, M. (2014). Jóvenes, ficción televisiva y videojuegos: espectáculo, tensión $y$ entretenimiento. Tendencias generales de consumo. En: Revista de Estudios de Juventud, $\mathrm{n}^{\circ}$ 106. Madrid: Instituto de la Juventud, pp. 77-91. Disponible en http://www.injuve.es/ sites/default/files/2014/47/publicaciones/5\%20 J\% C3\%B3venes \%20ficci\% C3\%B3n, \% 20 televisiva\%20y\%20videojuegos.pdf (Consultado el 16 de mayo de 2016).

-Espinosa, L. (2015). Medios de comunicación y prácticas discursivas: notas para el levantamiento de un estado actual. En: Poliantea, vol. 11, n²0. Bogotá: Institución Universitaria Politécnico Grancolombiano, pp.143-174. Disponible en https://dialnet.unirioja.es/servlet/ articulo?codigo $=5251685$ (Consultado el 16 de febrero de 2016).

-Ferguson, K. (2014). Everything is a Remix. Disponible en http://everythingisaremix.info/ (Consultado el 23 de mayo de 2014).

-Ferreiro,A. (2012). El consumidor de contenidos del Siglo XXI. Disponible en http://homelandz. com/el-consumidor-de-contenidos-del-siglo-xxi/
(Consultado el 10 de enero de 2016).

-Grandío, M. (2011). Redes y comunicación audiovisual. En: Noguera, J. M.; Martínez Polo, J. y Grandío, M. Redes sociales para estudiantes de Comunicación. Barcelona: Editorial UOC, pp.121-168.

-Hernández P. Juan \& Martinez D. Miguel (2017). Nuevos modelos de consumo audiovisual: los efectos del binge-watching sobre los jóvenes universitarios En: adComunica. Revista Científica de Estrategias, Tendencias e Innovación en Comunicación, n¹3. Castellón: Asociación para el Desarrollo de la Comunicación adComunica y Universitat Jaume I, 201-221. DOI: http://dx.doi. org/10.6035/2174-0992.2017.13.11.

- Longwell, T. (2014)Americans addicted to binge viewing, willing to pay extra for it. Disponible en http://streamdaily.tv/2014/04/30/americansaddicted-to-binge-viewing-willing-to-pay-extrafor-it/ (Consultado el 10 de enero de 2016)

- López Vidales, Nereida etal. (2012). Preferencia juvenil en nuevos formatos de televisión. Tendencias de consumo en jóvenes de 14 a 25 años. Proyecto de investigación realizado por el Observatorio del Ocio y el Entretenimiento Digital (OCENDI). Disponible en: http://www.ocendi. com/descargas/informetv_web.pdf (Consultado el 15 de mayo de 2016). 